\section{Little and large}

\section{Carole Jordan}

Atoms in Astrophysics.

Edited by P.G. Burke et al.

Plenum: 1983. Pp.350. \$49.50, £34.65.

THE interaction between atomic physics and astrophysics has its origins in work of the nineteenth-century spectroscopists, who used stellar spectra to establish empirical formulae to describe the series of lines observed. Thus began a fruitful dialogue - discoveries from astrophysical spectra stimulating atomic physics, atomic theory providing the means by which quantitative analyses and further astrophysical progress could be made. Since its foundation by H.S.W. Massey, this crossfertilization has been a recurring theme in the work of the atomic physics group at University College London. The group is now under the leadership of Professor Michael Seaton, whose sixtieth birthday is celebrated by the publication of this book.

The review articles in Atoms in Astrophysics are all by Seaton's colleagues, collaborators and past students. Naturally they emphasize those areas where Seaton has made his most significant contributions but in doing so they cover a wide range of topics. The early chapters on scattering theory by Burke and Eissner, and by Norcross, and the article on longrange potentials by Peach will interest

\section{Journals' review issue 1983}

On October 6th Nature will publish the third review supplement devoted to science journals. The two previous supplements (covering journals first published between January 1978 and May 1980, and June 1980 and May 1981) appeared in Nature 293, 341-369 (1981) and 299, 491-514 (1982).

Criteria for inclusion of a journal in the 1983 issue are that:

- the first number appeared, or the journal was re-titled, between June 1981 and May 1982;

- it is published at least three times a year;

- the main language used is English.

Broadly, periodicals of professional interest to scientists will be considered for review, with the exception of abstracts' journals. Journals appearing prior to June 1981 but after January 1978, and not covered in the previous issues, will also be considered.

Publishers and societies are invited to submit four sample issues of journals satisfying the above criteria, including the first and most recent numbers, to the Review Editor, Nature, 4 Little Essex St, London WC2R 3LF, England (London 8366633 ext 2570) as soon as possible. specialists in atomic theory. The later essays, which concentrate on applications to planetary nebulae and hot plasmas, will be of particular value to astrophysicists.

One cannot think of quantum defect theory, ably reviewed by Moores and Saraph, without being aware of Seaton's contributions over many years. The great potential of quantum defect theory was apparent even in the early work and it still finds an application in modern calculations associated with autoionization and dielectronic recombination, both of importance to astrophysicists. Dubau and Van Regemorter review applications of atomic theory to the interpretation of the spectra of hot plasmas in astrophysical sources and laboratory nuclear fusion devices. Since the early 1960 s observations from space in the extreme-ultra-violet and $X$-ray spectral regions have led to an increasing requirement for a wide range of atomic data. The progress of the atomic theory has in several instances been stimulated by discoveries made first from solar spectra. This close liaison between solar physics and atomic physics is now bearing fruit also in the context of the interpretation of EUV spectra of a wide range of astrophysical objects, obtained with the International Ultraviolet Explorer satellite. Seaton's own astrophysical interest has been mainly in planetary nebulae, discussed here by Flower. Seaton applied many of his early calculations of collisional excitation cross-sections to forbidden transitions in these low density nebulae.

It has always been Seaton's hope that simple methods of computing atomic data could be developed to encourage astronomers to make their own calculations of rates required. The UCL program SUPERSTRUCTURE is discussed by Nussbaumer and Storey. It is now used at several locations in Europe and the United States, and has gone some way to satisfy that aim. However, other approaches to the same concept continue to be required and the more general state of such programs and of data banks is discussed by Burke and Eissner.

The volume would not have been complete without the account by Bates of the historical development of research on the forbidden atomic lines in auroral spectra. It was in this context 30 years ago that Seaton applied his early calculation of excitation by electron impact. A sentence by Bates, if generalized beyond auroral spectra, expresses the importance of atomic physics to astrophysics - "The results that Seaton provided initiated the slow advance . . . from the promiscuity of qualitative reasoning to the discrimination of quantitiative reasoning". Atoms in Astrophysics sets out in a historical context some of the methods by which this has been achieved.

Carole Jordan is a Lecturer in Theoretical Physics at the University of Oxford.

\section{At the frontiers of simple systems}

\section{William Klemperer}

\section{Chemical Dynamics via Molecular}

Beam and Laser

Techniques.

By R.B. Bernstein.

Oxford University Press: 1982. Pp. 262.

Hbk £25, \$49.50; pbk £10.95, \$19.50.

R.B. Bernstein has brought many new methods and insights to the study of the dynamics of chemical reactions. This monograph is a most enjoyable transcription of his 1980 Hinshelwood lectures and conveys well their excitement. The lectures are a well balanced blend of experimental and theoretical methods used to probe, in essence, the non-statistical features that may occur in chemical reactions. This, then, is not a textbook of chemical dynamics; rather it is a tour guide to the frontiers of the subject.

Bernstein describes the methods and results of quantum state preparation of reactants and also quantum state analysis of products. A great deal of discussion is devoted to the most important question of vibrational energy selectivity in the rates of chemical reactions. The account of reactions whose dynamics can be treated by classical motion on a single potential surface follows a chapter on non-reactive scattering. The question of inversion, namely obtaining the potential from observation is treated in both of these chapters. There is, of course, also a chapter on the information theoretical approach, which provides the balance to detailed trajectories on a potential surface. All of the nine chapters are lucidly written and include upto-date reference lists of relevant review articles.

At this stage in the development of chemical dynamics, the emphasis in the subject is on simple systems. Most of the systems studied involve diatomic species, where there is a single rotational and a single vibrational quantum number. Studies examining the selectivity of reaction rates towards vibrational, rotational and translational energy can be systematically pursued, as Bernstein illustrates by using a number of well-chosen examples.

The lectures deal with low-pressure gasphase reactions of extreme simplicity, and the reader of this monograph will certainly gain knowledge about what is feasible in the investigation of the details of simple chemical reactions. However the book also provides insights which will be valuable in guiding the study of more complex systems. In this sense it should appeal to a wide audience.

William Klemperer is a Professor in the Department of Chemistry, Harvard L'niversity. 